\title{
Análise dos Sistemas de Informações de Hospitais
}

\author{
Antônio Artur de Souza \\ artur@face.ufmg.br \\ Thaísy Las-Casas de Macedo Amorim \\ thaisy@ufmg.br \\ Mariana Guerra \\ mariguerra@ufmg.br
}

\section{RESUMO}

No atual contexto brasileiro, as organizações necessitam obter informações que auxiliem no processo decisório. Os Sistemas de Informações (SIs) são instrumentos que fornecem informações úteis para esse processo. Entretanto, a maioria dos hospitais não utiliza sistemas que adequadamente orientem decisões e auxiliem o controle de atividades. Essa pesquisa objetivou principalmente analisar os Sls de um hospital filantrópico situado em Belo Horizonte/MG, denominado Alfa. Para tanto, buscou-se descrever o SI e verificar sua adequabilidade. Realizou-se também uma pesquisa complementar em outros três hospitais: Beta, Gama e Delta. Pelo estudo, observou-se que Alfa enfrenta dificuldades para controlar e organizar suas atividades operacionais e administrativas, além de dificuldades na implantação e gestão de Sls. Esses fatos também se comprovam pelo estudo complementar, que demonstrou que hospitais filantrópicos e não filantrópicos também têm dificuldades de implantar, adaptar e utilizar Sls.

Palavras-chave: Hospitais, Sistemas de Informações, Sistemas de Gestão de Custos

\section{INTRODUÇÃO}

No atual ambiente competitivo, as organizações necessitam de informações adequadas e acuradas para a tomada de decisão. $\mathrm{E}$ as informações sobre custos são consideradas cruciais para a prosperidade das organizações. Sem informações adequadas sobre custos, o gestor pode tomar decisões errôneas e ocasionar inclusive a falência da empresa. Nesse contexto, as organizações têm buscado continuamente meios confiáveis de obter essas informações. Os Sistemas de Informações (SIs) são instrumentos capazes de coletar dados e transformá-los em informações úteis para subsidiar o processo decisório (MOSCOVE et al., 2002).

Com a crescente competitividade entre as organizações, inclusive entre as não empresariais, uma administração eficaz e eficiente é também fundamental para os hospitais. A busca pelo menor custo, sem afetar a qualidade e a funcionalidade dos serviços, é um objetivo permanente das organizações que buscam sustentabilidade no mercado. Para que se encontre esse menor custo, as informações devem representar ao máximo a realidade vivenciada pela organização, tornando o processo decisório mais correto possível. Entretanto, grande parte das organizações hospitalares não faz uso de um sistema de custos e de informações financeiras que oriente e ofereça parâmetros para suas decisões administrativas e para o controle de atividades (ABBAS, 2001).

Nesse contexto, essa pesquisa teve como objetivo principal analisar os Sls de um hospital filantrópico situado na cidade de Belo Horizonte/MG, denominado nesse artigo de Hospital Alfa. Além desse estudo de caso, realizou-se uma pesquisa complementar sobre os Sls utilizados em outros três hospitais da cidade - hospitais Beta, Gama e Delta. Inicialmente, na seção 2, apresentam-se alguns aspectos sobre Sls. Na seção 3, apresentam-se informações sobre sistemas de gestão de custos e sobre Sistema de Informações Hospitalares (SIH). Na seção 4, descreve-se a metodologia da pesquisa. A análise dos resultados da pesquisa no hospital Alfa é apresentada na seção 5. Além disso, na seção 6, apresentamse as comparações dos Sls utilizados nos quatro hospitais pesquisados - Alfa, Beta, Gama e Delta. Finalmente, apresenta-se a conclusão na seção 7 .

\section{SISTEMA DE INFORMAÇÕES}

Segundo Bodnar e Hopwood (2002), Sistema de Informação (SI) é uma coleção de hardware e software utilizada para transformar dados em informações úteis. Para Moscove et al. (2002), um SI possui vários elementos inter-relacionados que coletam (entrada no sistema), manipulam e armazenam (onde se tem os processos), disseminam os dados e as informações (saídas do sistema) 
e que fornecem um mecanismo de feedback. Segundo Bürkle et al. (2001), a eficácia do SI consiste: (1) na acessibilidade do sistema aos usuários que realizam a entrada dos dados e aos que necessitam das informações disponibilizadas pelo sistema; e (2) na geração de informações precisas, confiáveis, completas, oportunas, flexíveis e objetivas. Além disso, deve-se considerar o fato de as restrições tecnológicas, humanas e organizacionais não prejudicarem a funcionalidade do SI, nem a qualidade das informações. Dessa forma, a classificação de SI varia bastante dependendo do princípio utilizado para distinguí-los. Stair (1998), por exemplo, divide os SI em quatro grandes grupos: Sistemas de Informações Transacionais (SIT), Sistemas de Informações Gerenciais (SIG), Sistemas de Apoio à Decisão (SAD) e Sistema Especialista (SE).

Os SIG, especificamente, asseguram a execução efetiva das estratégias empresariais, fornecendo informações periódicas sobre as operações e a produtividade a partir de bases de dados que são processadas de acordo com as necessidades do usuário. Esse sistema é destinado às atividades funcionais dos administradores e utilizado principalmente para planejamento e organização. Os Sistemas de Gestão de Custos (SGC), em especial, fazem parte do SIG.

Laudon e Laudon (1999) ainda classificam os sistemas em três tipos principais, de acordo com a responsabilidade assumida por seus usuários dentro da organização. O Sistema de Nível Operacional auxilia no trabalho de execução, de acompanhamento e de armazenamento das operações diárias da organização, dando suporte aos usuários que trabalham com os dados e o conhecimento. O Sistema de Nível Gerencial dá suporte à tomada de decisões, ao controle e ao monitoramento, utilizando dados da operação para permitir a obtenção de informações que possibilitem a gestão da organização. O Sistema de Nível Estratégico, por fim, utiliza dados de todos os sistemas, de forma processada e agregada para as decisões de mais alto nível na organização. Esses autores argumentam que nenhum sistema coordena as atividades de uma organização inteira sozinho. Usualmente, as organizações possuem diferentes tipos de Sls para enfocar diferentes níveis de problemas e diferentes funções dentro da organização. A Figura 1 ilustra esta situação com uma visão integrada do papel do Sl em uma organização, em que se demonstra o nível organizacional e os vários comandos (as gerências sênior e média, os trabalhadores do conhecimento e os funcionários de produção e serviços), assim como o problema organizacional (estratégico, tático, conhecimento e operacional). Na base da organização estão as várias áreas interligadas.

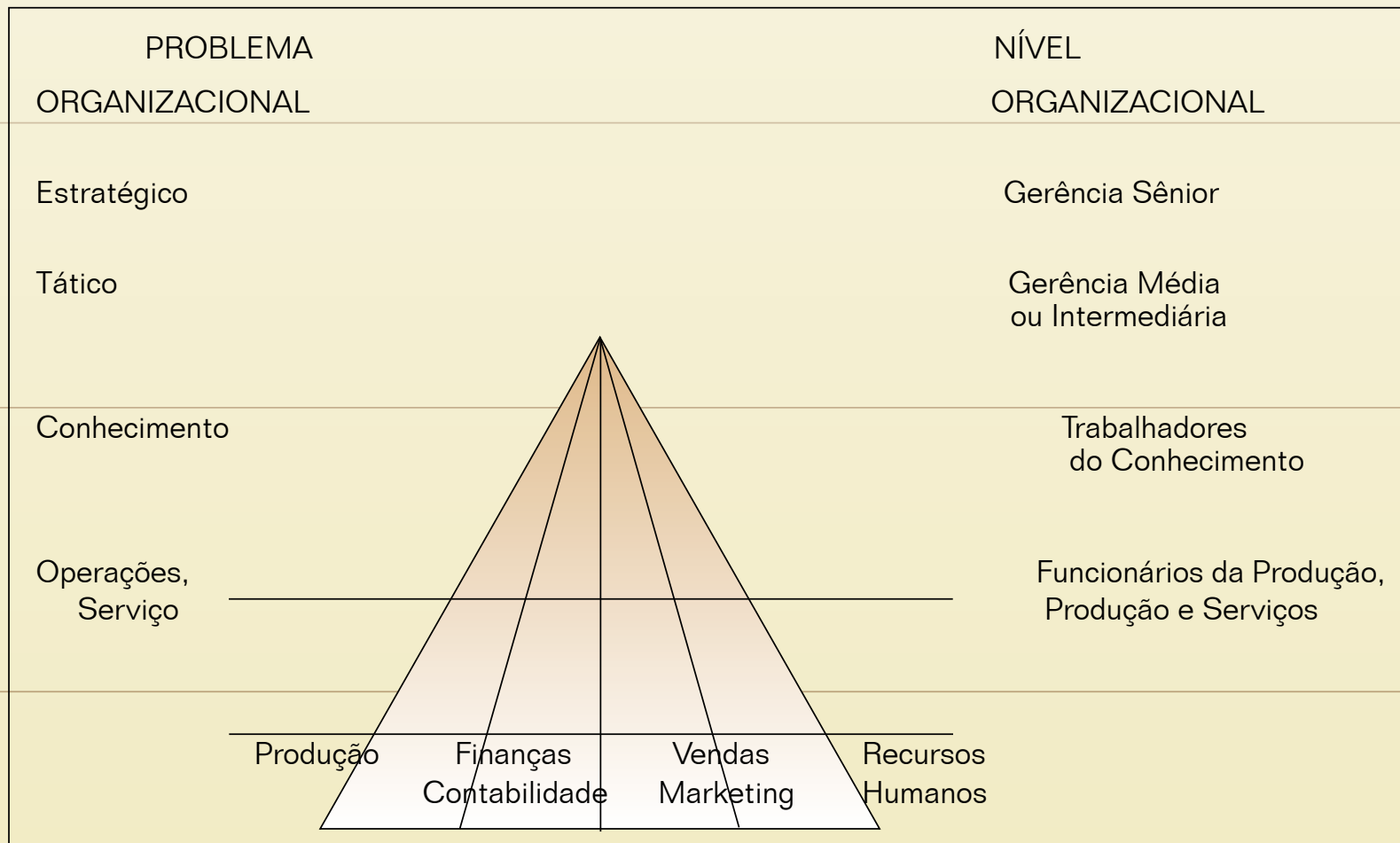

Figura 1 - Visão Integrada do papel dos Sls em uma organização FONTE: Laudon e Laudon (1999) 


\section{SISTEMA DE GESTÃO DE CUSTOS E SISTEMAS DE INFORMAÇÕES HOSPITALARES}

Para as organizações, a adoção dos principais aspectos teóricos e metodológicos apresentados na teoria da gestão de custos, aliada a um conjunto de recursos técnicos da informática, constituem os chamados SGC. Esses sistemas têm sido utilizados por organizações que primeiramente buscam o custeamento dos serviços e dos produtos, e em seguida o controle periódico dos resultados das atividades da organização por meio da mensuração do desempenho (COOPER; KAPLAN, 1992). Leone (2000) acredita que um SGC caracteriza-se por apresentar funções semelhantes a um centro processador de dados e de produção de informações destinadas aos diferentes níveis gerenciais da organização.

Toda a área de Tecnologia de Informações (TI), incluindo o SIG, tem por finalidade contribuir para o desenvolvimento de um SGC que minimize os fatores que dificultam a utilização adequada desses sistemas. Para Cooper e Kaplan (1992), esses fatores são a inadeqüabilidade do SIT relacionadas ao SGC, causando re-trabalho ou dificuldade de obtenção dos dados em relação aos controles internos; e os erros de alocação ou de rastreamento dos custos indiretos aos produtos ou serviços, devido à falta de SIT que possa dar suporte na identificação das medidas de alocação ou no rastreamento dos custos indiretos.

Os Sistemas de Informações Hospitalares (SIH), por sua vez, têm por objetivo suprir as necessidades operacionais dos diversos setores ou serviços existentes, bem como proporcionar integração entre os mesmos. A utilização de SIH decorre da necessidade de se organizar e se controlar o volume de informações e de processos executados em um hospital, que exigem um controle ágil e flexível de modo a evitar a repetição de tarefas e os desperdícios. Dada a própria natureza da assistência médico-hospitalar, estas organizações possuem características de ordem científica, econômica, social e política, cuja sistematização é muito difícil. Entretanto, como estratégia inicial para a implantação de um SIH, Johanston (1993) defende a necessidade de se avaliar a viabilidade de aquisição ou de criação de um sistema conforme os recursos existentes no próprio hospital e no seu mercado específico, a facilidade de interação do usuário com o sistema, assim como as informações possíveis de serem gerenciadas. Outro fator fundamental é a questão da interface entre os programas ou os módulos utilizados na organização.

\section{METODOLOGIA DE PESQUISA}

O método de pesquisa adotado no presente trabalho foi o estudo de caso. De acordo com Yin (2005), o estudo de caso é uma estratégia de pesquisa que contribui para a compreensão dos fenômenos individuais, organizacionais, sociais e políticos complexos, por intermédio de uma investigação ex post facto de eventos da vida real. As técnicas de coleta de dados utilizadas foram: pesquisa bibliográfica em livros, artigos, dissertações, teses e materiais disponíveis em sítios; observações diretas não participantes, entrevistas e consulta a arquivos e documentos. As entrevistas foram a principal fonte de dados para a realização desta pesquisa, complementada e/ou confirmada pelas demais técnicas de coleta de dados (pesquisa documental e observação direta não participante). Como sugere Yin (2005), também foi utilizado o protocolo de pesquisa. Este tem a finalidade de conduzir a coleta de dados e principalmente registrar as anotações de campo ao final de cada visita.

A pesquisa foi realizada em um hospital filantrópico, situado na cidade de Belo Horizonte/MG, tanto no setor responsável pela administração e controle do sistema de informação, como nos setores operacionais e de apoio, tais como farmácia, almoxarifado etc. Os dados primários foram obtidos a partir de visitas in loco, realizadas no segundo semestre de 2007. No presente artigo, esse hospital é denominado Alfa. Após essa pesquisa, outros três hospitais situados na cidade de Belo Horizonte/MG também foram estudados, entretanto de forma complementar. Essas organizações são denominadas no presente artigo de Beta, Gama e Delta. Os critérios abordados, fundamentados na pesquisa de Kuwabara (2003), serviram de base para comparação dessas organizações - Alfa, Beta, Gama e Delta. Para as organizações pesquisadas de forma complementar, buscou-se abordar apenas os aspectos qualitativos na análise do sistema: quais informações são acessíveis, qual a aceitabilidade e a adaptabilidade do SI por parte dos usuários, assim como a satisfação dos funcionários na utilização do sistema. O Quadro 1 apresenta os principais pontos abordados nas entrevistas e os objetivos específicos a serem alcançados em cada item pesquisado nas organizações estudadas. 


\begin{tabular}{|c|l|l|}
\hline Item & Questões & Objetivos \\
\hline 1 & $\begin{array}{l}\text { Você tem acesso facilitado ao } \\
\text { sistema? }\end{array}$ & Evidenciar limitações do SI \\
\hline 2 & $\begin{array}{l}\text { Os Sls atendem às suas necessidades } \\
\text { e às de sua área? }\end{array}$ & Detectar disponibilidade de informações \\
\hline 3 & $\begin{array}{l}\text { Você é treinado quando uma nova } \\
\text { solução é disponibilizada no seu setor? }\end{array}$ & Detectar adaptabilidade e aceitabilidade do SI \\
\hline 4 & $\begin{array}{l}\text { Como você classifica a qualidade do } \\
\text { sistema? }\end{array}$ & Detectar satisfação do usuário \\
\hline
\end{tabular}

FONTE: Adaptado de Kuwabara (2003)

\section{SISTEMA DE INFORMAÇÕES E SISTEMA DE GESTÃO DE CUSTOS NO HOSPITAL FILANTRÓPICO ALFA}

O setor responsável pela administração e pelo controle do SI do Hospital Alfa conta com um funcionário responsável pela operação dos computadores, que realiza atividades de suporte em software e em hardware. O sistema operacional que Alfa utiliza em seus servidores é o Linux. Nas estações de trabalho, nos setores operacionais, o Hospital trabalha com o sistema operacional Windows, nas versões 98 ou XP. O SIH utilizado, denominado Sistema de Gestão Hospitalar (SGH) Windows é fornecido pela SPDATA e está interligado em rede em todas as áreas desse hospital, e por meio desse, gerencia-se grande parte dos setores. Além disso, a organização não possui um sistema único que forneça todos as informações e todos os serviços necessários, pois a administração de Alfa considera que, por ser muito complexo, o Hospital não conseguiria ter o controle por meio de um único sistema. Dessa forma, Alfa conta com um sistema específico para o Controle de Infecção Hospitalar, além do software do Sistema Único de Saúde (SUS) - único convênio desse hospital - para gerar as contas dos pacientes.

No Hospital Alfa, o SGH Windows é utilizado para controlar os setores de internação, farmácia, estoque, faturamento, e para emitir contas de pacientes que devem ser entregues ao SUS. Com esse sistema SGH Windows - realizam-se também atividades do setor financeiro, de controle de patrimônio, de compras, assim como de Contabilidade. Além disso, através do Serviço de Apoio à Diagnose e Terapia (SADT), é realizada a gestão do laboratório, do raio-x e de todos os exames que o paciente possa precisar para fazer um diagnóstico. Entretanto, o Hospital Alfa possui setores que ainda não utilizam nenhum SI, que são os setores de lavanderia, de limpeza e a Central de Materiais Esterilizados (CME). Quanto à alimentação do sistema, essa é feita em todos os setores que o utilizam, conforme a necessidade.

Quando funcionários do Hospital precisam de informações específicas, que serão temporárias e que não estão agregadas a esse sistema, utilizam os programas Access ${ }^{\circledR}$ ou Microsoft ${ }^{\circledR}$ Excel. De acordo com a administração de Alfa, as informações geradas pelo SGH Windows são claras e objetivas e o acesso a essas informações finais é específico para usuários do setor de origem. Apenas o setor de Contabilidade tem acesso a todas as informações. Além disso, os relatórios só podem ser obtidos de acordo com a necessidade de cada setor específico e com as informações que esse mesmo setor fornece. A administração de Alfa acredita na necessidade desses relatórios para a tomada de decisões, e, nesse processo, utilizam as informações históricas disponível no sistema.

Quanto à confiabilidade, a administração do Hospital Alfa pontua com 80\% de satisfação o sistema utilizado atualmente. Entretanto, a causa apontada para a não totalidade dessa pontuação não se relaciona à deficiência do próprio sistema, mas à deficiência de processos, da política da organização e da forma como o sistema é alimentado. Segundo a administração, o trabalho no Hospital é muito dinâmico, dessa forma, nem sempre os funcionários conseguem alimentar o sistema de forma ideal ou necessária, o que pode comprometer a satisfação dos usuários. Portanto, não há confiabilidade nas informações constantes nesse relatório. Como pontos fortes do sistema, a administração de Alfa afirma que a segurança e a tecnologia usadas são de ótima qualidade. Uma facilidade encontrada é que, apesar de terem comprado o sistema como um "pacote fechado", as modificações que são necessárias especificamente para o Hospital são feitas pela empresa fornecedora. Entretanto, o ponto fraco encontrado é que justamente essas alterações feitas para um hospital serão repassadas para todos os hospitais que utilizam o sistema, e algumas vezes essa atualização não é interessante para todos.

Quanto ao estado dos computadores, $10 \%$ das máquinas já possuem mais de dois anos de uso. Os últimos computadores comprados possuem um ano de uso. Por isso, a administração de Alfa planeja comprar mais dez computadores no próximo ano. Especificamente, o SGC não é um sistema à parte nesse Hospital. O próprio SGH Windows fornece as informações sobre custos para os usuários do sistema. No Hospital Alfa, segundo seus administradores, o sistema SGH Windows forneceria as informações necessárias e com facilidade sobre os custos, porém o sistema não é alimentado de forma a gerar relatórios ou informações 
precisas. Os usuários não têm informações sobre o custo de um procedimento, ou por paciente, ou qualquer custo específico. O sistema utilizado em Alfa possui ferramentas para coletar dados e gerar informações, assim como para controlar os custos. Oferece custos médios por procedimento, por centro de custos, por departamento, ou global. Entretanto, a maneira como os usuários alimentam o SI não é suficiente.

\section{COMPARAÇÃO ENTRE OS SISTEMAS DE INFORMAÇÕES DO HOSPITAL FILANTRÓPICO ALFA COM OUTROS TRÊS HOSPITAIS COMPLEMENTARES}

\subsection{Hospital Beta}

O Hospital Beta também é uma organização filantrópica. Para essa análise, foram realizadas entrevistas nos setores de compras, de farmácia, de almoxarifado e de Contabilidade. Segundo os funcionários responsáveis pelo setor de almoxarifado, o sistema gera pedidos automáticos à medida que o estoque diminui, não sendo necessário realizar requisições. Esse fato demonstra que o SI utilizado no Hospital Beta nesse setor é um sistema integrado e que executa tarefas programadas por seus usuários e atividades não-rotineiras. Segundo os funcionários, no setor de compras, o acesso às informações de estoque é muito limitado, uma vez que é permitido aos seus usuários apenas gerar ordens de compra e relatórios com dados do custo total da compra. Além disso, o acesso às informações de estoque por é permitido somente aos setores de almoxarifado e de farmácia. Ao setor de compras não são disponibilizadas essas informações. Devido a essa restrição, o setor de almoxarifado não exerce controle em relação ao setor de compras. Esse fato explicita certa limitação à integração e à acessibilidade do sistema.

Em relação ao setor de farmácia, existe um sistema específico de controle de estoque. Entretanto, a requisição de inclusão ou de compra de estoques é realizada de forma manual por um funcionário específico do Hospital. Apesar disso, destaca-se que esse sistema atende ao consumo de todos os almoxarifados dessa organização. Segundo os funcionários desse setor, esse sistema é centralizado e permite realizar atividades de entrada e de saída de dados, de controle de validade e de controle psicotrófico. Entretanto, apesar de ser um sistema abrangente, há alguns problemas de acesso e de aceitabilidade por parte dos funcionários do setor. Destaca-se ainda que, segundo funcionário de farmácia, não é possível afirmar se esse sistema é integrado ao setor de Contabilidade. Entretanto, sabe-se que há seis relatórios enviados à Contabilidade que são baseados em informações do sistema específico do setor de farmácia. Semestralmente, esse setor de Beta faz um inventário, a fim de "acertar" os valores financeiros da Contabilidade. Entretanto, deve-se destacar um ponto negativo desse sistema. Segundo funcionários do setor de farmácia, algumas vezes, o sistema apresenta problemas e ocorre alteração no controle de estoque. Especificamente, houve um erro no sistema em que foi realizada uma duplicação de todas as entradas então efetuadas. Além disso, após essa ocorrência, o sistema apresentou estoque nulo. Em relação ao setor de almoxarifado, os usuários apresentam significativa satisfação em relação à utilização do sistema. Destaca-se que o controle de patrimônio é realizado pela Contabilidade, em um sistema próprio. $\mathrm{E}$, em relação a esse último setor, os usuários desenvolvem ferramentas para a captação dos custos. $\mathrm{O}$ sistema específico do setor de Contabilidade utiliza centro de custos, que são automáticos. Além disso, os consumos já possuem percentuais fixos para o centro de custos. Pelo SI utilizado, é possível realizar um controle mensal e outro semestral em cada ano. Entretanto, esse sistema não possui controle de folha de pagamentos informatizado. Segundo seus usuários, o sistema é defasado e a organização do Hospital Beta pensa em implementar outro SI no setor. Além disso, a Contabilidade também utiliza o sistema que o SUS disponibiliza para gerar as contas vinculadas aos serviços prestados. Dessa forma, pode-se concluir que os sistemas utilizados por Beta não são interligados. Segundo seus usuários, os sistemas não fornecem todas as informações necessárias e há dificuldade em acessá-las. Por esses motivos, há certa insatisfação com a utilização dos sistemas disponibilizados nos diversos setores do Hospital. Apesar disso, os funcionários de Beta afirmam que os Sls utilizados apresentam facilidade de adaptação.

\subsection{Hospital Gama}

O Hospital Gama é uma organização privada. Para a análise da utilização dos Sls empregados, foram realizadas entrevistas nos setores de compras, de farmácia, de almoxarifado e de CME. Especificamente para o setor de farmácia, segundo os funcionários de Gama, o lançamento de kits disponibilizados pelo Hospital com materiais para realizar certos procedimentos é efetuado via sistema, através de código de barras. Além disso, esses kits são automaticamente vinculados à conta do paciente, e tanto a secretária do setor, quanto a funcionária específica da farmácia possuem acesso. Apesar dessas facilidades, segundo seus usuários, o sistema é bastante criticado e pode ser denominado de "burro". Outras atividades realizadas via SI no setor de farmácia são: agendamento de cirurgias e requisição de materiais. Para tanto, o sistema cria etiquetas para medicamentos e kits. Porém, os lotes dos medicamentos registrados nas etiquetas não conferem com os lotes informados pelo sistema, fato que dificulta o controle. Por isso, a conferência dos medicamentos que estão registrados é realizada manualmente por uma funcionária específica. 
Para o setor de almoxarifado, pelo sistema utilizado, é possível elaborar gráficos de curva que evidenciam os dados do sistema de custo utilizado - no caso o sistema de custeio baseado em atividade (ABC). Além disso, é possível elaborar relatórios sobre o consumo por período ou por grupo de serviços. Apesar disso, o sistema do almoxarifado não dispõe do controle sobre estoque mínimo e máximo. O SI utilizado, apesar de possibilitar o controle de $100 \%$ do estoque, não é utilizado em sua amplitude, uma vez que Gama está em fase de adaptação. O sistema do almoxarifado foi recentemente implementado, e seus usuários ainda precisam se adequar aos recursos e às informações disponíveis. Por essas características, pode-se observar que os sistemas utilizados por Gama são integrados nas diversas áreas da organização. Além disso, possibilita o setor de almoxarifado controlar 100\% dos estoques do hospital, caracterizando o sistema como significativamente abrangente em relação a disponibilidade de informações. De forma bastante distinta, os funcionários do setor de CME do Hospital não realizam as atividades via SI. Dessa forma, as requisições não são informatizadas, e por isso, são realizadas manualmente através de registro em papéis. Já para o setor de compras, o SI utilizado ainda é novo. Dessa forma, os usuários pouco se adaptaram ao sistema e as requisições de compras ainda são realizadas manualmente.

Segundo a administração de Gama, esse sistema oferece adaptações (upgrade) aos contadores. Além disso, é possível, através do SI, controlar o estoque do setor de compras, porém essa atividade ainda é realizada manualmente. Apesar da necessidade de adaptação e de adequação do sistema ao setor, os funcionários de compras apresentam-se bastante satisfeitos com a implementação e a progressiva utilização do SI. Segundo observações, é possível concluir que, em relação aos Sls utilizados pelos setores pesquisados do Hospital Gama, alguns usuários de informações estão satisfeitos com o sistema implementado. Entretanto, as atividades de parte dos setores pesquisados não são desenvolvidas por meio da utilização de Sls. Segundo funcionários entrevistados, diversas atividades ainda são realizadas de forma manual e não estão integradas a outros setores essenciais ao desenvolvimento de específico serviço ou atividade. Os setores que utilizam SI ainda estão em fase de adaptação aos recursos e às informações disponíveis no sistema.

\subsection{Hospital Delta}

O Hospital Delta é um hospital privado e sem fins lucrativos. Para a coleta de dados foram realizadas entrevistas nos setores de serviços gerais, lavanderia, bloco cirúrgico, farmácia, enfermaria, almoxarifado central e suprimentos (compras). O setor de serviços gerais é responsável por limpeza, manutenção e transporte de todo o Hospital. Neste setor existe um almoxarifado de material exclusivo para a limpeza, portanto, separado do almoxarifado central. Dessa forma, o material utilizado para a limpeza é retirado do almoxarifado central através de requisições. Especificamente, o controle dos serviços e dos materiais utilizados na manutenção e no transporte é feito através de ordens de serviços preenchidas manualmente. Segundo funcionários do setor, não é utilizado um sistema integrado para a realização das atividades. Entretanto, o responsável pelo setor utiliza as planilhas do software Excel ${ }^{\circledR}$, onde estão descritos todos os serviços e os materiais utilizados.

Já o setor de lavanderia presta serviços para os setores de enfermaria e do bloco cirúrgico, sendo 70\% de suas atividades dedicadas ao primeiro setor. Segundo funcionários da lavanderia, não é utilizado nenhum tipo de SI informatizado, e todo o material utilizado é retirado no almoxarifado central através de requisições. De forma semelhante, o setor de bloco cirúrgico também não utiliza um SI específico. Todos os recursos empregados na realização dos procedimentos são anotados manualmente em fichas de prontuários dos pacientes e posteriormente repassados para o setor de faturamento. Além disso, o agendamento de cirurgias é controlado manualmente em formulários do Hospital. Delta possui duas farmácias, sendo uma central e outra exclusiva para o bloco cirúrgico, chamada farmácia satélite. Todo o controle é feito pela farmácia central que utiliza um sistema integrado com a satélite. Além desse sistema, as farmácias utilizam formulários eletrônicos para as transferências de medicamentos. O setor de enfermaria, por sua vez, possui um sistema em que são cadastrados os pacientes que utilizam os serviços prestados nesse setor. Destaca-se que a portaria de Delta é considerada o principal usuário das informações disponibilizadas pelo SI do setor de enfermaria.

O almoxarifado central utiliza um sistema em que estão cadastrados os produtos ou materiais utilizados pelos demais setores do Hospital. A retirada desses é autorizada por meio de requisições de cada setor. O almoxarifado central relaciona-se diretamente ao setor de suprimentos, porém não há um SI integrado. Dessa forma, para solicitar a compra de algum produto, o almoxarifado utiliza formulários manuais, chamados de ordens de compra. O setor de suprimentos (compras), por sua vez, utiliza um SI específico, em que são cadastrados os pedidos de compras, fornecedores, orçamentos etc. Destaca-se que este sistema é integrado com o setor financeiro. Por fim, o setor de radiologia não utiliza um SI específico. Por isso, o material utilizado é anotado manualmente na ficha dos pacientes. Além disso, o serviço de radiologia é solicitado através de formulário também manual específico desse setor. O que se percebe no Hospital Delta é que algumas atividades são realizadas de forma manual e não integradas a outros setores essenciais ao desenvolvimento de específico serviço ou atividade. Por outro lado, alguns setores encontram-se satisfatoriamente favorecidos com Sls, que podem disponibilizar informações também a outros setores. 


\subsection{Comparação entre os hospitais Alfa, Beta, Gama e Delta}

Como mencionado, o Hospital Alfa é uma organização filantrópica. Os Sls utilizados para coletar dados e transformá-los em informações úteis para o processo de decisório são relativamente precários e não abrangem, de forma integrada, diversos setores do Hospital. A adaptabilidade do SI para as áreas pesquisadas também é limitada. Entretanto, apesar de alguns funcionários dizerem-se insatisfeitos com o sistema implementado, a maioria encontra-se satisfeito com o SI utilizado. Além disso, por meio do estudo, pode-se concluir que há aceitabilidade dos sistemas. O Hospital Beta também é uma organização filantrópica, entretanto de capacidade operacional (número de leitos) significativamente maior do que Alfa. Apesar disso, os sistemas utilizados por Beta também não são interligados. Segundo seus usuários, os Sls não fornecem todas as informações necessárias e há dificuldade em acessá-las. Dessa forma, pode-se observar que a aceitabilidade do sistema é ruim. Por esses motivos, há certa insatisfação com a utilização dos Sis disponibilizados nos diversos setores do Hospital. Entretanto, os funcionários de Beta afirmam que os Sls utilizados apresentam facilidade de adaptação.

Gama é uma organização privada e com o menor número de leitos disponíveis, além disso, não presta serviços ao SUS. Segundo estudo, alguns usuários de informações nesse Hospital estão satisfeitos com o sistema implementado. Algumas atividades desenvolvidas são integradas a dois setores, ou seja, há informações que podem ser utilizadas por até dois setores. Apesar disso, o que se observou no estudo do SI é que não há integração que abrange os diversos setores. Segundo funcionários entrevistados, diversas atividades são realizadas de forma manual. Nos setores em que há disponibilidade de um SI, observou-se certa abrangência em relação às informações fornecidas. Apesar de os usuários encontrarem-se em fase de adaptação ao SI, esses dizem estar satisfeitos com o sistema.

O Hospital Delta, por sua vez, é uma organização privada e sem fins lucrativos. Por meio da análise dos Sls utilizados, pode-se perceber que alguns usuários de informações realizam atividades de forma manual e não integradas a outros setores essenciais ao desenvolvimento de específico serviço ou atividade. Nos setores em que há disponível um SI, os usuários encontram-se satisfatoriamente favorecidos pelo sistema.

Conforme descrito na literatura pesquisada, a utilização de SIH decorre da necessidade das organizações hospitalares de se organizar e controlar o volume de informações e de processos executados. Isso exigem um controle ágil e flexível, de modo a evitar repetição de tarefas e desperdícios. Entretanto, o que se observa, segundo o estudo, é que os hospitais ainda não se adaptaram as novas tecnologias empregadas, assim como não há adequação dos Sls a gestão e a operação das atividades executadas. Por meio da pesquisa e segundo literatura utilizada, pode-se observar que os hospitais enfrentam dificuldades quando têm de tomar decisões para implantação, aquisição, renovação e gestão dos sistemas ou quando precisam compatibilizar as atividades diárias com a constante evolução da tecnologia e das técnicas médicas (MADI, 1998). Segundo esse autor e conforme observado na pesquisa, de modo geral, as dificuldades encontradas estão presentes tanto nas organizações hospitalares públicas, quanto nas privadas.

\section{CONCLUSÃO}

O estudo de caso realizado no Hospital Alfa possibilitou (1) levantar vários dados referentes à organização e (2) discutir questões importantes referentes ao SGC utilizado. Concluiu-se que os Sls disponíveis nos diversos setores de Alfa são adequados somente para satisfazer às necessidades de informações dos setores individualmente. Entretanto, setores distintos não conseguem acessar as informações relativas às demais áreas. Apesar de constatar satisfação parcial dos usuários, o que se observou é que não há integração entre os Sls utilizados em Alfa. As informações disponibilizadas a um determinado setor só podem ser acessadas/utilizadas por esse mesmo setor. Além disso, a disponibilidade de informações não é satisfatória, e não atende satisfatoriamente as necessidades dos usuários. O que se observou como principal causa da limitação em se utilizar ou se adaptar ao SI foi o fato de que a implantação do sistema em Alfa ainda é recente. Os usuários provavelmente não se familiarizaram aos recursos e às acessibilidades disponíveis nos sistemas.

O Hospital Beta também é uma organização filantrópica, entretanto de capacidade operacional maior do que o primeiro Hospital. De forma semelhante a Alfa, os Sls não são integrados e também há dificuldade em se acessar as informações disponíveis. Entretanto, Beta difere-se de Alfa em relação a satisfação dos usuários, pois na primeira organização há significativa desaprovação dos Sls utilizados. Gama, por sua vez, é uma organização privada, e alguns usuários de informações estão satisfeitos com o sistema implementado no setor. Apesar da limitação de acesso às informações de usuários de áreas distintas, há disponibilidade de informações no setor de origem. Entretanto, diversas atividades são realizadas de forma manual e não integradas. Essa organização assemelha-se bastante ao Hospital Delta, que é uma organização privada, sem fins lucrativos, com menor número de leitos disponíveis e que não presta serviços aos SUS.

Diversas atividades também são manuais no Delta. Nos setores em que há disponível um SI, os usuários de informações estão satisfeitos com o sistema implementado. Apesar disso, não é possível afirmar que os usuários obtêm todas as informações necessárias. 
Pela pesquisa, pôde-se observar que o Hospital Alfa enfrenta dificuldades quando têm de tomar decisões para implantação, aquisição, renovação e gestão dos sistemas ou quando precisam adequar o desenvolvimento e controle das atividades à constante evolução da tecnologia e das técnicas médicas. Esse fato também se comprova nas demais organizações pesquisadas. O estudo complementar demonstrou que organizações de diferentes capacidades operacionais ou classificação empresarial também apresentam dificuldades para implantação e adaptação de Sls.

\section{REFERÊNCIAS BIBLIOGRÁFICAS}

ABBAS, K. Gestão de custos em organizações hospitalares. 155 f. Dissertação (Mestrado em Engenharia de Produção), Universidade Federal de Santa Catarina, Florianópolis, 2001.

BODNAR, G.H.; HOPWOOD, W. S. Accounting information systems. 8. ed. New Jersey: PrenticeHall, 2002.

BÜRKLE, T. et al. Evaluation of clinical information systems. what can be evaluated and what cannot? Journal of Evaluation in Clinical Practice. v. 7, n. 4, p. 373-385, 2001.

COOPER, R.; KAPLAN, R. S. The design of cost management systems: text, cases and readings. New Jersey: Prentice-Hall, 1992.

JOHANSTON, H. Sistemas de informação hospitalar: presente e futuro. Revista Informédica, v.1, n.2, p.5-9, 1993. Disponível em: <http://Informação \PALAVRAS\%20UTEIS \Johanston,\%20F>. Acesso em: 14 nov. 2007.

KUWABARA, C. C. T. Avaliação do sistema de informação: estudo de caso de um hospital escola público. Dissertação (Mestrado em Administração) - Universidade Estadual de Maringá, Maringá, 2003.

LAUDON, K. C.; LAUDON, J. P. Sistemas de informação com internet. 4. ed. Rio de Janeiro: LTC, 1999.

LEONE, G. Custos: planejamento, implantação e controle. 3. ed. São Paulo: Atlas, 2000.

MADI, E. D. T. Planejamento para evolução do ambiente de tecnologia de informação do Hospital das Clínicas da Unicamp. 174f. Dissertação (Mestrado em Informática) - Instituto de Informática da Pontifícia Universidade Católica de Campinas, Campinas, 1998.

MOSCOVE, S. A. et al.Critical Sucess Factors for Implementing an ERP System in a University Enviroment: a case study from the Australian HES. 201f. Dissertação (Mestrado). Faculty of Engineering an Information Technology, Griffith University, Austrália, 2002.

STAIR, R. M. Sistemas de Informação. Rio de Janeiro: LTC, 1998.

YIN, R. K. Estudo de caso: planejamento e métodos. 3. ed. Porto Alegre: Bookman, 2005. 